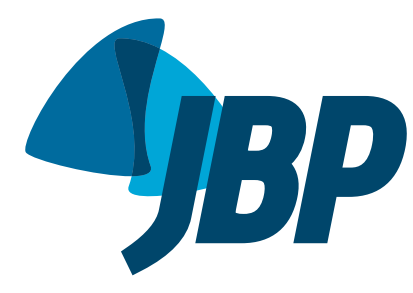

1. Universidade Federal do Rio de Janeiro, Rio de Janeiro (RJ) Brasil.

2. Departamento de Radiologia, Radiologia Anchieta - Hospital Anchieta, Taguatinga (DF) Brasil.

3. Departamento de Radiologia, Instituto D'Or de Pesquisa e Educação, Rio de Janeiro (RJ) Brasil.

4. Departamento de Radiologia Santa Casa de Porto Alegre, Porto Alegre (RS) Brasil.

5. Multimagem Diagnósticos, Goiânia (GO) Brasil.

6. Departamento de Radiologia, Universidade Federal do Paraná, Curitiba (PR) Brasil.

7. Universidade Federal da Bahia, Salvador (BA) Brasil.

Submitted: 1 March 2017.

Accepted: 18 June 2017.

Study carried out at the Universidade

Federal do Rio de Janeiro

Rio de Janeiro (RJ) Brasil.

\section{Niemann-Pick disease type B: HRCT assessment of pulmonary involvement}

\author{
Heloisa Maria Pereira Freitas ${ }^{1}$, Alexandre Dias Mançano², \\ Rosana Souza Rodrigues ${ }^{1,3}$, Bruno Hochhegger ${ }^{4}$, \\ Pedro Paulo Teixeira e Silva Torres ${ }^{5}$, Dante Escuissato ${ }^{6}$, \\ Cesar Augusto Araujo Neto7, Edson Marchiori ${ }^{1}$
}

\begin{abstract}
Objective: To analyze HRCT findings in patients with Niemann-Pick disease (NPD) type $B$, in order to determine the frequency of HRCT patterns and their distribution in the lung parenchyma, as well as the most common clinical characteristics. Methods: We studied 13 patients ( 3 males and 10 females) aged 5 to 56 years. HRCT images were independently evaluated by two observers, and disagreements were resolved by consensus. The inclusion criteria were presence of abnormal HRCT findings and diagnosis of NPD type B confirmed by histopathological examination of a bone marrow, lung, or liver biopsy specimen. Results: The most common clinical findings were hepatosplenomegaly and mild to moderate dyspnea. The most common HRCT patterns were smooth interlobular septal thickening and ground-glass opacities, which were both present in all patients. Intralobular lines were present in 12 patients (92.3\%). A crazy-paving pattern was observed in 5 patients (38.4\%), and areas of air trapping were identified in only 1 case (7.6\%). Pulmonary involvement was bilateral in all cases, with the most affected area being the lower lung zone. Conclusions: Smooth interlobular septal thickening, with or without associated ground-glass opacities, in patients with hepatosplenomegaly is the most common finding in NPD type B.
\end{abstract}

Keywords: Niemann-Pick diseases; Tomography, X-ray computed; Lung diseases.

\section{INTRODUCTION}

Niemann-Pick disease (NPD) is a rare autosomal recessive hereditary disease characterized by acid sphingomyelinase deficiency, which causes accumulation of sphingomyelin, especially in the tissues of the reticuloendothelial system. ${ }^{(1-4)}$ Symptoms are due to accumulation of vacuolated lipid-filled macrophages, known as Niemann-Pick cells, in several organs, such as the liver, spleen, bone marrow, lung, and central nervous system. ${ }^{(2,4-6)}$

NPD is divided into six subtypes, from A to $F$, depending on the organs affected and the severity of the clinical course. (2) In subtype B, an enzymatic deficiency causes chronic visceral involvement, characterized by hepatosplenomegaly and lung and bone marrow involvement, without significant neurological deficit, with pulmonary involvement being one of the leading causes of morbidity and mortality. ${ }^{(2-4)}$ The presence of hepatosplenomegaly associated with specific HRCT findings, such as interlobular septal thickening, groundglass opacities, intralobular lines, and a crazy-paving pattern, constitutes important information that indicates NPD as a likely diagnosis. ${ }^{(3,4,7)}$ Although a presumptive diagnosis can be made on the basis of clinical history and radiological findings, laboratory studies-measurement of sphingomyelinase activity in peripheral leukocytes together with fibroblast cell culture or analysis of bone marrow biopsy specimens revealing accumulation of lipid-filled macrophages (sea-blue histiocytes)-are definitive for the diagnosis. ${ }^{(8)}$ In the present study, we analyzed HRCT scans from 13 patients with NPD type $B$, all of whom had histopathological confirmation. The objectives of the present study were to describe the most common HRCT findings and the major clinical and epidemiological characteristics observed in these patients.

\section{METHODS}

This was a descriptive, retrospective, observational study of chest HRCT scans from 13 patients with NPD type $B$. These scans were randomly gathered from personal contact with radiologists and pulmonologists from eight different institutions, located in six Brazilian states. The diagnoses were suspected on the basis of a clinical history of hepatosplenomegaly associated with chest HRCT findings. All diagnoses were confirmed by biopsy.

Pulmonary changes were assessed using HRCT. There was no standardization of HRCT assessment because multiple institutions were involved, with HRCT scans being performed according to the protocol used at each facility. However, all HRCT scans were performed at end-inspiration, using 1- to 2-mm-thick slices, at 5- to

Correspondence to:

Edson Marchiori. Rua Thomaz Cameron, 438, Valparaiso, CEP 25685-120, Petrópolis, RJ, Brasil.

Tel.: 5524 2249-2777. Fax: 5521 2629-9017. E-mail: edmarchiori@gmail.com

Financial support: None. 
10-mm intervals, from the apex to the hemidiaphragm, with patients in the supine position. Images were reconstructed on a $512 \times 512$ pixel matrix, digitized, and photographed, for assessment of the lung fields, at window widths of $1,000-1,500 \mathrm{HU}$ and window levels of -650 to $-750 \mathrm{HU}$. For assessment of the mediastinum, window width was set to 350-500 $\mathrm{HU}$, and the window center level was set to 40-60 HU. There was no administration of intravenous iodinated contrast medium. All HRCT scans were independently evaluated by two observers, and disagreements were resolved by consensus.

The scans were analyzed for the following characteristics: lesion distribution relative to their predominance in the upper or lower halves of the lungs; and pattern of changes (interlobular septal thickening, intralobular lines, ground-glass opacities, a crazy-paving pattern, and other pulmonary or extrapulmonary changes). Interlobular septal thickening was defined as the presence of linear opacities delimiting secondary pulmonary lobules. Intralobular lines were defined as thin linear images within the secondary pulmonary lobule. When numerous, they have a fine, lacy appearance. Ground-glass opacity was defined as increased pulmonary attenuation, with preserved bronchial and vascular margins. A crazy-paving pattern was defined as ground-glass opacities superimposed on interlobular septal thickening. Air trapping was defined as decreased lung parenchymal attenuation, apparent mainly as lower-than-usual density, and absence of volume reduction, both of which result from excessive accumulation of gas in the parenchyma due to partial or total airway obstruction. The criteria for defining these findings are those provided by the glossary of terms for thoracic imaging from the Fleischner Society ${ }^{\left({ }^{(9)}\right.}$ and the terminology used is that presented in the consensus statement on terminology developed by the Imaging Department of the Brazilian Thoracic Association. ${ }^{(10)}$

\section{RESULTS}

We evaluated 13 patients with NPD type B with pulmonary involvement, $10(76.9 \%)$ of whom were female and $3(23.1 \%)$ of whom were male. The patients ranged in age from 5 to 56 years, with a mean age of 27 years. In the sample, there were four pairs of siblings. All patients had hepatosplenomegaly, which was painless in $6(46.1 \%)$. Eight $(61.5 \%)$ had had hepatosplenomegaly since childhood. From a respiratory standpoint, 8 patients (61.5\%) were asymptomatic, and $5(38.4 \%)$ had dyspnea.

The HRCT findings in descending order of frequency were smooth interlobular septal thickening ( $n=13$; $100.0 \%$; Figure 1$)$; ground-glass opacities ( $n=13$; $100.0 \%)$; intralobular lines ( $n=12 ; 92.3 \%)$; a crazypaving pattern ( $\mathrm{n}=5 ; 38.4 \%$; Figure 2$)$; and areas of air trapping ( $n=1 ; 7.6 \%$; Figure 3$)$. The ground-glass opacities were focal in 10 patients (76.9\%) and diffuse in $3(23.1 \%)$. The pulmonary involvement was bilateral in all of the 13 cases studied, predominantly affecting the lower lobes, with no laterality predominance.
At the time of HRCT, 6 patients were 16 years of age or younger (ranging in age from 5 to 16 years, with a mean of 11.7 years) and 7 were 22 years of age or older (ranging in age from 22 to 56 years, with a mean of 40.3 years). Extensive ground-glass opacities were identified in 4 of the 6 patients who were 16 years of age or younger, but in only 1 of the 7 patients who were 22 years of age or older. In addition, of the 5 patients who had a crazy-paving pattern, 4 were 16 years of age or younger.

Hepatosplenomegaly was an important clinical finding in our series of patients with NPD type B. Splenomegaly was more marked than hepatomegaly. Three patients also underwent abdominal CT. Curiously, the 3 patients had hypodense splenic nodules. These nodules were not biopsied.

\section{DISCUSSION}

In our sample, 10 patients (76.9\%) were female and $3(23.1 \%)$ were male. McGovern et al. ${ }^{(11)}$ reviewed 59 patients with a diagnosis of NPD type B. Gender prevalence was $53 \%$ male (31 patients) and $47 \%$ female (28 patients). This disagreement is probably due to the limited number of patients studied by us.

In our study, ages ranged from 5 to 56 years, with a mean age of 27 years. Six patients were less than 20 years of age. Our findings were relatively similar

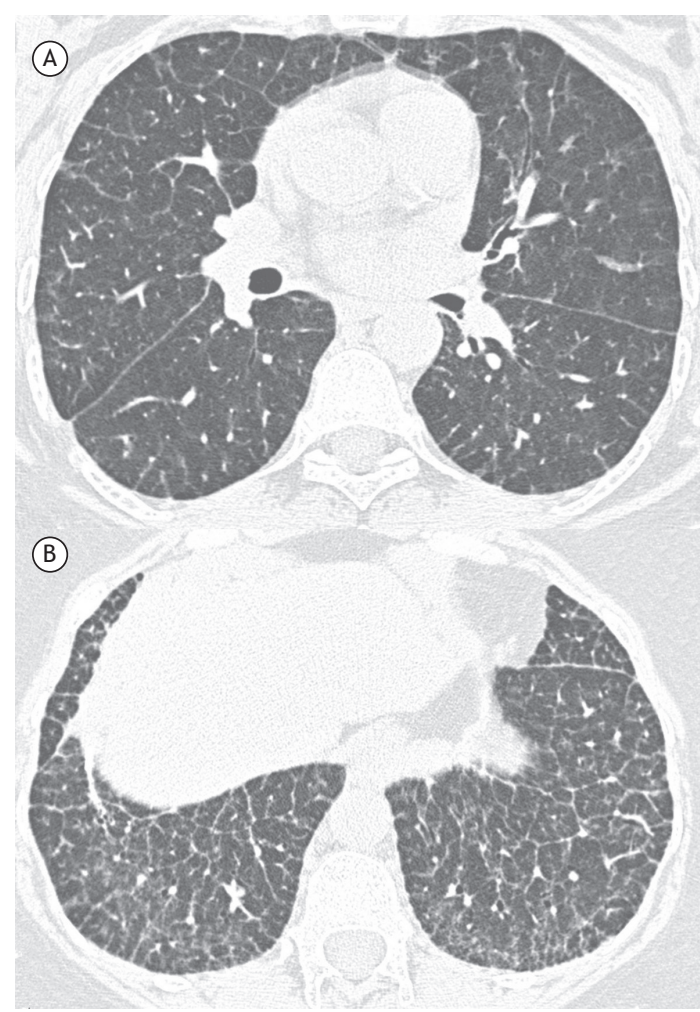

Figure 1. Twenty-eight-year-old female patient. Chest HRCT slices (lung window) from the lower lung zones. Note smooth interlobular septal thickening in both lungs (predominantly in the lower lobes), intralobular lines, and small foci of ground-glass opacity. 


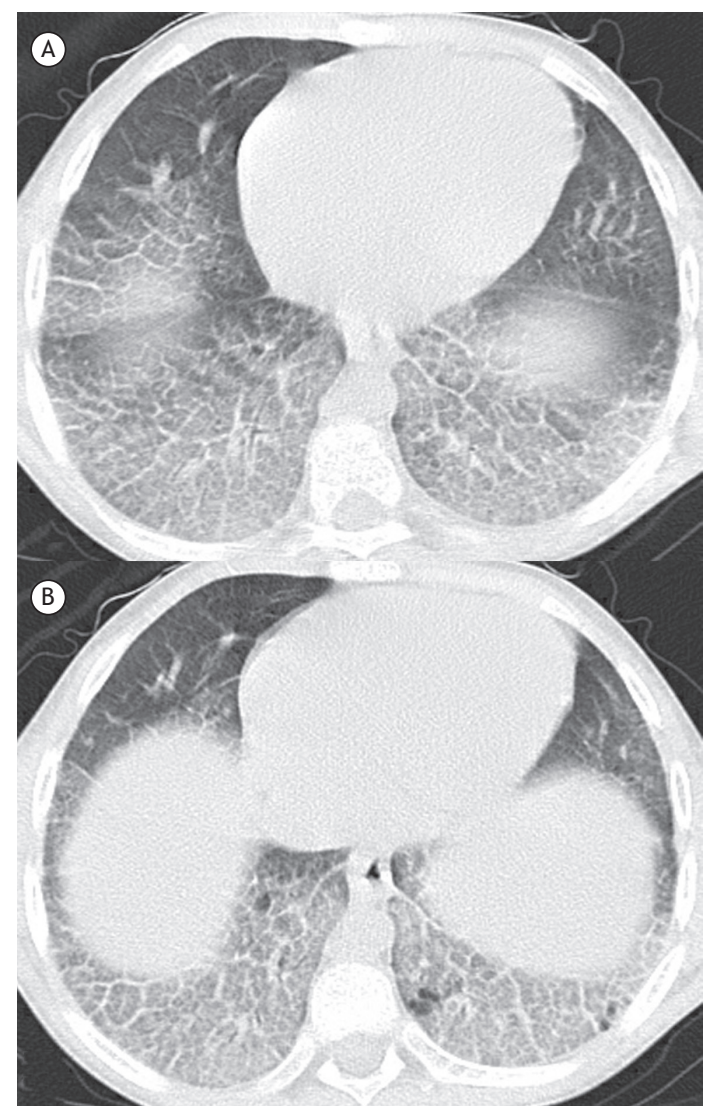

Figure 2. Sixteen-year-old male patient. Chest HRCT slices (lung window) from the lower lung zones. Note smooth interlobular septal thickening in both lungs (predominantly in the lower lobes), intralobular lines, and ground-glass opacities with a crazy-paving pattern.
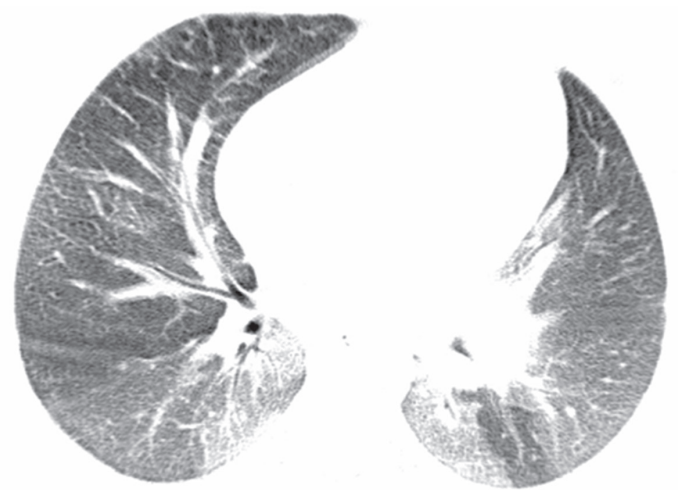

Figure 3. Fourteen-year-old female patient. HRCT scan of the chest. Note ground-glass opacities predominantly in the lower lobes, interspersed with areas of air trapping in the left lower lobe.

to data found in the literature. With regard to age at diagnosis, studies have shown that NPD type B has been reported in patients in different age groups, although it is most commonly observed in those less than 20 years of age. ${ }^{(12)}$ It is difficult to determine with precision the age group predominantly affected by NPD type B; however, the mean age at symptom onset is 5-7 years, and diagnostic suspicion is generally raised also in childhood, around age 10 years, with the detection of hepatosplenomegaly. ${ }^{(12)}$

Most patients initially present with hepatosplenomegaly (with the volume of the spleen being more marked than that of the liver), interstitial lung disease, and dyslipidemia. The absence of neurological involvement is one of the characteristics of NPD type $\mathrm{B} .{ }^{(11)}$ Laboratory and biochemical tests may reveal thrombocytopenia; abnormal liver function tests; high levels of lowdensity lipoprotein, very low-density lipoprotein, and triglycerides; and low levels of high-density lipoprotein and sphingomyelinase. ${ }^{(11,13)}$

In our sample, all patients had hepatosplenomegaly. The predominant respiratory symptom was mild to moderate dyspnea, in 5 patients (38.4\%). Eight patients $(61.5 \%)$ were asymptomatic from a respiratory standpoint. In the literature, the most commonly reported clinical finding is hepatosplenomegaly, present in approximately $75 \%$ of patients, followed by bleeding, dyspnea, pulmonary infections, and joint pain. There have also been reports of growth retardation in adolescence. ${ }^{(11)}$ Survival to adulthood occurs frequently, since the disease runs a chronic course, developing slowly and exhibiting a wide range of severity of symptoms and clinical findings. $(11,12,14)$ The disease rarely progresses to liver failure. ${ }^{(11,15)}$

NPD type B is familial in approximately one third of cases, given that it is an autosomal recessive hereditary disease. ${ }^{(16,17)}$ In our sample, we studied four pairs of siblings. However, the incidence of hereditary association may be greater, since the families of the remaining patients were not adequately investigated to exclude other familial cases.

The diagnosis can be confirmed by bone marrow, lung, or liver biopsy findings of accumulation of finely vacuolated, foamy, lipid-filled macrophages. ${ }^{(11,13)}$ In the lung, these macrophages accumulate in the alveolar septa, bronchial walls, and pleura. ${ }^{(6,13)}$ In our sample, the diagnosis was confirmed by biopsy in all patients. The patients, because of their different institutions of origin, were not followed according to a pre-established protocol. Therefore, biopsies of various sites were performed at the discretion of the attending physician. Of the 13 patients, 5 underwent lung biopsy, 4 underwent liver biopsy, and 4 underwent bone marrow biopsy.

The most common HRCT patterns in our sample were interlobular septal thickening and ground-glass opacities, which were both present in the 13 cases $(100.0 \%)$. The ground-glass opacities were focal (in $76.9 \%$ ) and diffuse (in $23.1 \%$ ). The second most common pattern was intralobular lines, present in 12 of our patients (92.3\%). A crazy-paving pattern was found in 5 patients (38.4\%). It should be noted that, as was the case in our study, interlobular septal thickening and ground-glass opacities can coexist in the same patient without, however, causing a crazypaving pattern. Crazy-paving refers exclusively to the 
pattern of interlobular septal thickening superimposed on ground-glass opacities. These findings corroborate those reported in the literature. ${ }^{(1,3,11,16)}$ Areas of air trapping were observed in only 1 patient (7.6\%). We found no previous reports of air trapping associated with NPD. Although it is not possible to state that there is a direct relationship between air trapping and NPD, Baldi et al.,(2) reporting the finding of lung cysts in NPD, suggested that a possible mechanism would be macrophage migration into the bronchiolar lumen, leading to a check-valve mechanism and resulting in air trapping.

With regard to possible differences in HRCT findings between children and adults, 6 patients in our sample were 16 years of age or younger at the time of HRCT and 7 were 22 years of age or older. Extensive groundglass opacities were identified in 4 of the 6 patients who were 16 years of age or younger, but in only 1 of the 7 patients who were 22 years of age or older. In addition, of the 5 patients who had a crazy-paving pattern, 4 were 16 years of age or younger. We found no explanation in the literature for these differences observed in our sample.

NPD is a rare disease, and literature data on imaging tests are mostly found in case reports, there being very few studies focusing on the assessment of HRCT findings from series of patients. The most commonly reported $\mathrm{HRCT}$ findings in the literature are smooth interlobular septal thickening and intralobular lines, predominantly in the lower lobes of the lungs, and focal or diffuse ground-glass opacities, also predominantly in the lower lung zones. $(2,4,8,13,16)$ A crazy-paving pattern has also been reported. ${ }^{(3,4)}$ In the literature, the changes seen on CT most commonly affect the lower lobes, bilaterally. The same distribution was observed in our sample.

Smooth interlobular septal thickening can be observed in a large number of venous, lymphatic, or infiltrative diseases, the most common of which is pulmonary edema. Knowledge of the different causes of this pattern can be useful in preventing diagnostic errors. In addition, although the causes of this pattern are often indistinguishable by radiological assessment, differences in the distribution of lesions in the lungs, associated radiological findings, patient history, and clinical presentation can often be useful in suggesting the appropriate diagnosis. ${ }^{(18,19)}$

The major differential diagnoses of crazy-paving are due to acute or chronic causes. The major acute conditions that cause crazy-paving are pulmonary edema; ARDS; acute infections, such as pneumocystosis, leptospirosis, and H1N1 pneumonia; and diffuse alveolar hemorrhage. The major chronic conditions that cause crazy-paving are invasive adenocarcinoma, lipoid pneumonia, lymphangitic carcinomatosis, and alveolar proteinosis. ${ }^{(20-23)}$

The changes histopathologically correspond to the presence of foamy macrophages in the interlobular septa and some degree of interstitial fibrosis, resulting in an appearance of septal thickening. The same cells involving the alveoli are associated with ground-glass opacities. . $^{(2,13,15,24)}$

Hepatosplenomegaly is the most important clinical finding in NPD type $\mathrm{B}^{(11,25)}$ with splenomegaly being more marked than hepatomegaly. ${ }^{(6,11)}$ Splenomegaly may be associated with the presence of intraparenchymal nodules or masses, which have an echogenic appearance on ultrasonography and low attenuation values on $\mathrm{CT}_{\text {, }}$ as well as varied signal intensity on magnetic resonance imaging. Splenic nodules in NPD type B histologically consist of dilated sinuses containing lipid-filled macrophages (Niemann-Pick cells), demonstrated by Giemsa stain. (16) However, the nodules can also be associated with the presence of hemangiomas, ${ }^{(16,26)}$ areas of infarction, and lymphomas. ${ }^{(14,27)}$ In our sample, all patients had hepatosplenomegaly, 3 of whom (23.7\%) had hypodense splenic nodular lesions, which were revealed on HRCT. These lesions were not biopsied. The association of diffuse pulmonary infiltration and hepatosplenomegaly can be observed in a number of diseases of varying etiologies, such as infectious diseases (tuberculosis, fungal diseases, malaria, and schistosomiasis), neoplastic diseases (lymphoma and leukemia), metabolic diseases (amyloidosis and Gaucher's disease), autoimmune diseases (rheumatoid arthritis and systemic lupus erythematosus), and others (sarcoidosis, histiocytosis, hemosiderosis, etc.).(28,29) However, marked interlobular septal thickening associated with a history of hepatosplenomegaly since childhood is highly suggestive of storage disease. Therefore, the major differential diagnosis is Gaucher's disease. Although pulmonary involvement is uncommon in Gaucher's disease, clinical and imaging findings may be very similar, and, frequently, differential diagnosis is possible only on the basis of biopsies or enzymatic studies. $^{(16,30)}$

Our study had some limitations. The study was retrospective and observational. The analysis of some cases was cross-sectional, with the progression and possible complications of NPD not being assessed. The HRCT scans were performed according to the protocol used at each institution involved in the study. However, we do not believe that this variation had an impact on the results. Despite these limitations, we did not find in the literature any case series focusing on the study of CT findings from NPD type B patients and including as many cases as our study (the PubMed database was searched using the term "Niemann-Pick type B" in combination with "computed tomography").

In conclusion, the predominant HRCT patterns found in the patients with NPD type B were, in descending order of frequency, smooth interlobular septal thickening, ground-glass opacities, intralobular lines, and a crazy-paving pattern. A finding of smooth septal thickening in the lower lobes, associated with hepatosplenomegaly, especially in young patients, should raise the suspicion of NPD type B. 


\section{REFERENCES}

1. Ahuja J, Kanne JP, Meyer CA, Pipavath SN, Schmidt RA, Swanson $\mathrm{JO}$, et al. Histiocytic disorders of the chest: imaging findings. Radiographics. 2015;35(2):357-70. https://doi.org/10.1148/ rg.352140197

2. Baldi BG, Santana AN, Takagaki TY, Fujita C, Kairalla RA, Carvalho CR. Lung cyst: an unusual manifestation of Niemann-Pick disease. Respirology. 2009;14(1):134-6. https://doi.org/10.1111/j.14401843.2008.01352.x

3. Mendelson DS, Wasserstein MP, Desnick RJ, Glass R, Simpson W, Skloot G, et al. Type B Niemann-Pick disease: findings at chest radiography, thin-section $\mathrm{CT}$, and pulmonary function testing. Radiology. 2006;238(1):339-45. https://doi.org/10.1148/ radiol.2381041696

4. Rodrigues R, Marchiori E, Müller NL. Niemann-Pick disease: highresolution $\mathrm{CT}$ findings in two siblings. J Comput Assist Tomogr. 2004;28(1):52-4. https://doi.org/10.1097/00004728-20040100000008

5. Guillemot N, Troadec $\mathrm{C}$, de Villemeur TB, Clément A, Fauroux B. Lung disease in Niemann-Pick disease. Pediatr Pulmonol. 2007;42(12):1207-14. https://doi.org/10.1002/ppul.20725

6. Simpson WL Jr, Mendelson D, Wasserstein MP, McGovern MM. Imaging manifestations of Niemann-Pick disease type B. AJR Am J Roentgenol. 2010;194(1):W12-9. https://doi.org/10.2214/ AJR.09.2871

7. Hendriksz CJ. Inborn errors of metabolism for the diagnostic radiologist. Pediatr Radiol. 2009;39(3):211-20. https://doi. org/10.1007/s00247-008-1072-x

8. Mendes MS, Portela FX, Reis RC, Castro JD, Garcia JH, Holanda MA. Liver transplantation in a patient with Niemann-Pick disease and pulmonary involvement. J Bras Pneumol. 2012;38(2):269-71. https:// doi.org/10.1590/S1806-37132012000200018

9. Hansell DM, Bankier AA, MacMahon H, McLoud TC, Müller $\mathrm{NL}$, Remy J. Fleischner Society: glossary of terms for thoracic imaging. Radiology. 2008;246(3):697-722. https://doi.org/10.1148/ radiol.2462070712

10. Silva $\mathrm{Cl}$, Marchiori E, Souza Júnior AS, Müller NL; Comissão de Imagem da Sociedade Brasileira de Pneumologia e Tisiologia. Illustrated Brazilian consensus of terms and fundamental patterns in chest CT scans. J Bras Pneumol. 2010;36(1):99-123. https://doi. org/10.1590/S1806-37132010000100016

11. McGovern MM, Wasserstein MP, Giugliani R, Bembi B, Vanier MT, Mengel E, et al. A prospective, cross-sectional survey study of the natural history of Niemann-Pick disease type B. Pediatrics. 2008;122(2):e341-9. https://doi.org/10.1542/peds.2007-3016

12. Castañón Martínez R, Fernández-Velilla Peña M, González Montaño MV, Gómez Carrera L, Torres Sánchez MI. Lung affectation in an adult patient with Niemann-Pick disease, type B. Arch Bronconeumol. 2012;48(6):213-5. https://doi.org/10.1016//.arbr.2011.07.009

13. Nicholson AG, Florio R, Hansell DM, Bois RM, Wells AU, Hughes $P$, et al. Pulmonary involvement by Niemann-Pick disease. A report of six cases. Histopathology. 2006;48(5):596-603. https://doi. org/10.1111/j.1365-2559.2006.02355.x

14. Ferretti GR, Lantuejoul S, Brambilla E, Coulomb M. Case report. Pulmonary involvement in Niemann-Pick disease subtype B: CT findings. J Comput Assist Tomogr. 1996;20(6):990-2. https://doi. org/10.1097/00004728-199611000-00023

15. Muntaner L, Galmés A, Chabás A, Herrera M. Imaging features of
type-B Niemann-Pick disease. Eur Radiol. 1997;7(3):361-4. https:// doi.org/10.1007/s003300050167

16. von Ranke FM, Pereira Freitas HM, Mançano AD, Rodrigues RS, Hochhegger B, Escuissato D, et al. Pulmonary Involvement in Niemann-Pick Disease: A State-of-the-Art Review. Lung 2016;194(4):511-8. https://doi.org/10.1007/s00408-016-9893-0

17. Harzer K, Beck-Wödl S, Bauer P. Niemann-Pick disease type C: new aspects in a long published family - partial manifestations in heterozygotes. JIMD Rep. 2014;12:25-9. https://doi. org/10.1007/8904_2013_240

18. Griese M, Brasch F, Aldana VR, Cabrera MM, Goelnitz U, Ikonen $E$, et al. Respiratory disease in Niemann-Pick type C2 is caused by pulmonary alveolar proteinosis. Clin Genet. 2010;77(2):119-30. https://doi.org/10.1111/j.1399-0004.2009.01325.x

19. Rossi SE, Erasmus JJ, Volpacchio M, Franquet T, Castiglioni T, McAdams HP. "Crazy-paving" pattern at thin-section CT of the lungs: radiologic-pathologic overview. Radiographics. 2003;23(6):1509-19. https://doi.org/10.1148/rg.236035101

20. Marchiori E, Zanetti G, Hochhegger B. Interlobular septal thickening. J Bras Pneumol. 2016;42(2):161. https://doi.org/10.1590/S180637562015000000294

21. Hochhegger B, Schumacher Neto R, Marchiori E. Crazy-paving pattern. J Bras Pneumol. 2016;42(1):76. https://doi.org/10.1590/ S1806-37562016000000275

22. De Wever W, Meersschaert J, Coolen J, Verbeken E, Verschakelen JA. The crazy-paving pattern: a radiological-pathological correlation. Insights Imaging. 2011;2(2):117-132. https://doi.org/10.1007/s13244010-0060-5

23. Johkoh $T$, Itoh $H$, Müller $N L$, Ichikado $K$, Nakamura $H$, Ikezoe J, et al. Crazy-paving appearance at thin-section CT: spectrum of disease and pathologic findings. Radiology. 1999;211(1):155-60. https://doi. org/10.1148/radiology.211.1.r99ap10155

24. von Ranke F, Zanetti G, Hochhegger B, Marchiori E. Infectious diseases causing diffuse alveolar hemorrhage in immunocompetent patients: a state-of-the-art review. Lung. 2013;191(1):9-18. https:// doi.org/10.1007/s00408-012-9431-7

25. Wasserstein MP, Desnick RJ, Schuchman EH, Hossain S, Wallenstein S, Lamm C, et al. The natural history of type B NiemannPick disease: results from a 10-year longitudinal study. Pediatrics. 2004;114(6):e672-7. https://doi.org/10.1542/peds.2004-0887

26. Benedetti E, Proietti A, Miccoli P, Basolo F, Ciancia E, Erba PA, et al. Contrast-enhanced ultrasonography in nodular splenomegaly associated with type B Niemann-Pick disease: an atypical hemangioma enhancement pattern. J Ultrasound. 2009;12(3):85-92.

27. Omarini LP, Frank-Burkhardt SE, Seemayer TA, Mentha G, Terrier F. Niemann-Pick disease type C: nodular splenomegaly. Abdom Imaging. 1995;20(2):157-60. https://doi.org/10.1007/BF00201528

28. vom Dahl S, Mengel E. Lysosomal storage diseases as differential diagnosis of hepatosplenomegaly. Best Pract Res Clin Gastroenterol. 2010;24(5):619-28. https://doi.org/10.1016/j.bpg.2010.09.001

29. Elsayes KM, Narra VR, Mukundan G, Lewis JS Jr, Menias CO Heiken JP. MR imaging of the spleen: spectrum of abnormalities. Radiographics. 2005;25(4):967-82. https://doi.org/10.1148/ rg.254045154

30. Gülhan B, Ozçelik U, Gürakan F, Güçer $S$, Orhan D, Cinel G, et al. Different features of lung involvement in Niemann-Pick disease and Gaucher disease. Respir Med. 2012;106(9):1278-85. https://doi. org/10.1016/j.rmed.2012.06.014 